\title{
Doses delivered by portal imaging quality assurance in routine practice of adjuvant breast radiotherapy worth to by monitored and compensated in some cases
}

\author{
Sami Kefs ${ }^{1,2}$, Jean-Yves Giraud ${ }^{1,2}$, Julie Naud ${ }^{1}$, Isabelle Henry ${ }^{1}$, Isabelle Gabelle-Flandin ${ }^{1}$, \\ Jacques Balosso ${ }^{1,2,3}$, Abdulhamid Chaikh ${ }^{4}$, Camille Verry ${ }^{1,2}$ \\ ${ }^{1}$ Department of Cancerology-Radiotherapy, University Hospital Grenoble-Alpes, Grenoble, France; ${ }^{2}$ INSERM SRMR Team (Synchrotron Radiation \\ and Medical Research), ESRF, Grenoble, France; ${ }^{3}$ University Grenoble-Alpes, Grenoble, France; ${ }^{4}$ LPCC - Laboratoire de physique corpusculaire de \\ Caen CNRS, France
}

Correspondence to: Abdulhamid Chaikh. LPCC - Laboratoire de physique corpusculaire de Caen, F-14000 Caen, France.

Email: abdulhamedc@yahoo.com.

\begin{abstract}
Background: Imaging, in radiotherapy, has become a routine tool for repositioning of the target volume at each session. The repositioning precision, currently infracentimetric, evolves along with the irradiation techniques. This retrospective study aimed to identify practices and doses resulting from the use of high energy planar imaging (portal imaging) in daily practice.

Methods: A retrospective survey of portal images (PIs) was carried out over 10 years for 2,403 patients and for three linacs (1 Elekta SLi, 2 Varian Clinac) for postoperative mammary irradiations. Images were taken using a standardized number of monitor units (MU) for all patients. Due to the variable sensitivities of the detectors and the possibility of adjustment of the detector-patient distance, the number of MU were $3 ; 2$ and 1 respectively, for Elekta SLi ${ }^{\circledR}$, Clinac $600^{\circledR}$ and Clinac $2100^{\circledR}$. Then, a representative cumulated dose was calculated in simplified reference conditions ( $5 \mathrm{~cm}$ depth, beam of $10 \mathrm{~cm} \times 10 \mathrm{~cm}, 6 \mathrm{MV}$ ), considering the total number of images taken during the whole treatment course. The consistency between the representative doses and the actual absorbed doses received by the patients was verified by simulating a series of typical cases with the treatment plan dose calculation system.

Results: The delivered doses differ significantly between the three linacs. The mean representative dose values by complete treatment were 0.695; 0.241 and $0.216 \mathrm{~Gy}$, respectively, for SLi, Clinac 600 and Clinac 2100. However, 15 patients were exposed to a dose $>2$ Gy with a maximum dose of 5.05 Gy. The simulated doses were very similar to the representative doses.

Conclusions: A significant dose delivery was highlighted by this study. These representative doses are presently communicated weekly to the radiation oncologist for the radiation protection of their patients. Moreover, they should be taken into account in a possible study of long-term stochastic risks.
\end{abstract}

Keywords: Portal imaging; breast cancer; dose; radiotherapy

Submitted Dec 10, 2019. Accepted for publication Mar 22, 2021.

doi: 10.21037 /qims-19-1031

View this article at: http://dx.doi.org/10.21037/qims-19-1031

\section{Introduction}

The use of ionizing radiation in medicine is not legally dose limited but must respect two fundamental obligations for the radioprotection of the patients which are the justification and the optimization (1). In radiotherapy, optimization, in the sense of radiation protection, is represented by the reduction of all the doses that don't contribute to the dose at the target volume. Thus, the control of additional doses 
administered as part of quality control imaging is justified.

The increasingly demanding precision of advanced irradiation techniques (IMRT, stereotactic, etc.) requires a verification of the target repositioning at each session, or several times per session when very large doses per fraction are delivered (Cyberknife, important hypofractionation, etc.). The precision of this repositioning is today always infracentimetric, even millimetric and therefore requires the use of medical imaging. So, the INCa (French National Cancer Institute) in the Accreditation Criteria for External Radiation Therapy stated that "Patient positioning is checked at least once a week by an imaging device included in the treatment machine" (2).

Although these are low doses compared to radiotherapy, the progression of cancer cure rates, especially in young patients, raises the problem of the long-term stochastic effects of radiotherapy. Many financial and technical efforts will be made in the future to reduce the impact of therapeutic doses in these patients (proton therapy), the control of repositioning imaging doses must participate in this process.

Moreover, in borderline situations of tolerance dose, or even exceeding these doses, the dose contribution of the positioning imaging can deliver a supplement of proven and deleterious toxicity as it was highlighted during the overirradiations crisis of Epinal Hospital in France (3).

There are different types of repositioning imaging system in radiotherapy. Planar imaging that can be performed in two ways: the high energy portal image (PI) with the therapeutic beam [megavoltage (MV)] and the low energy imaging in $\mathrm{kV}$ using $\mathrm{X}$-ray tubes located in the treatment room, as the ExacTrac ${ }^{\circledR}$ system of $\mathrm{BrainLab}^{\circledR}$, allow pretreatments and intra-treatment readjustments on bone structures or radiopaque fiducials (gold grains). This last type of $\mathrm{kV}$ registration is carried out daily for the accuracy of the treatment, while the portal imaging, linked to the treatment machine, is mandatory and carried out weekly for quality assurance. However, portal imaging can be used at each session if no $\mathrm{kV}$ imaging is available. The other type is volume imaging type megavoltage computed tomography (MVCT), cone beam computed tomography (CBCT) or others, which are transverse tomographic images made with onboard beams sources, thus linked to the treatment machine. For accuracy requirements, these imaging procedures, when available, are used daily throughout the treatment.

The images are therefore numerous since almost daily for several weeks of treatment. However, in some cases of hypo-fractionation or radiosurgery, several or many images are carried out in shorter periods of time during the same session.

Moreover, in addition to repositioning imaging, radiotherapy patients will always be given radiological or nuclear medicine examinations and anyway a dosimetry CT scan with an acquisition of greater or lesser thickness (0.625 to $5 \mathrm{~mm}$ ) according to the anatomical locations to study. All these imaging procedures should also be taken into account in a global management of radiation protection of patients.

Accounting of the doses delivered by imaging is difficult because they depend on many parameters: the configuration of the devices (source-patient and patient-detector distance), the energy of the beams, the type of acquisition, etc. In addition, depending on the energy used, the dose delivered will predominantly affect the skin or deep tissues.

Finally, it is important to distinguish 2 fundamental points: on the one hand, the distinction between the locally absorbed dose (in gray) and the effective dose (in sievert), the latter represents the integrated whole-body dose in respect of stochastic risk assessment; on the other hand, the distinction between the two types of image acquisition. Planar images deliver a rather heterogeneous dose in a limited volume, which decreases when the detector is approached; while volume imaging, CT type, has an almost uniform distribution of dose in a larger study area. Therefore, for similar delivered dose in the air at the isocenter, the real patient's exposure will not be the same.

Several studies evaluated the dosimetric consequences using portal imaging in clinical practice. This includes megavoltage electronic portal imaging (MV-EPI), kilovoltage digital radiography $(\mathrm{kV} \mathrm{DR})$, tomotherapy MVCT, megavoltage cone-beam CT (MV-CBCT) and kilovoltage cone-beam CT (kV-CBCT). They compared the dose for organs in breast, brain, prostate and pelvis using different beam energy. They showed that, for daily positioning, the organ dose can be significantly reduced depending on $\mathrm{kV}$ or $\mathrm{MV}$ imaging. The delivered dose from imaging procedures contributes to the total organ dose. In addition, the dose from portal imaging should also be considered. A more in-depth analysis of the literature is given in the discussion (4-9).

However, the originality of our work is to calculate these doses on a real-life scale including, as far we may know, the largest homogeneous cohort, comparing three accelerators for exactly the same technique. Moreover, beyond the simple observation we provided a real practice study, as it gave way to establish routine information for radiation 


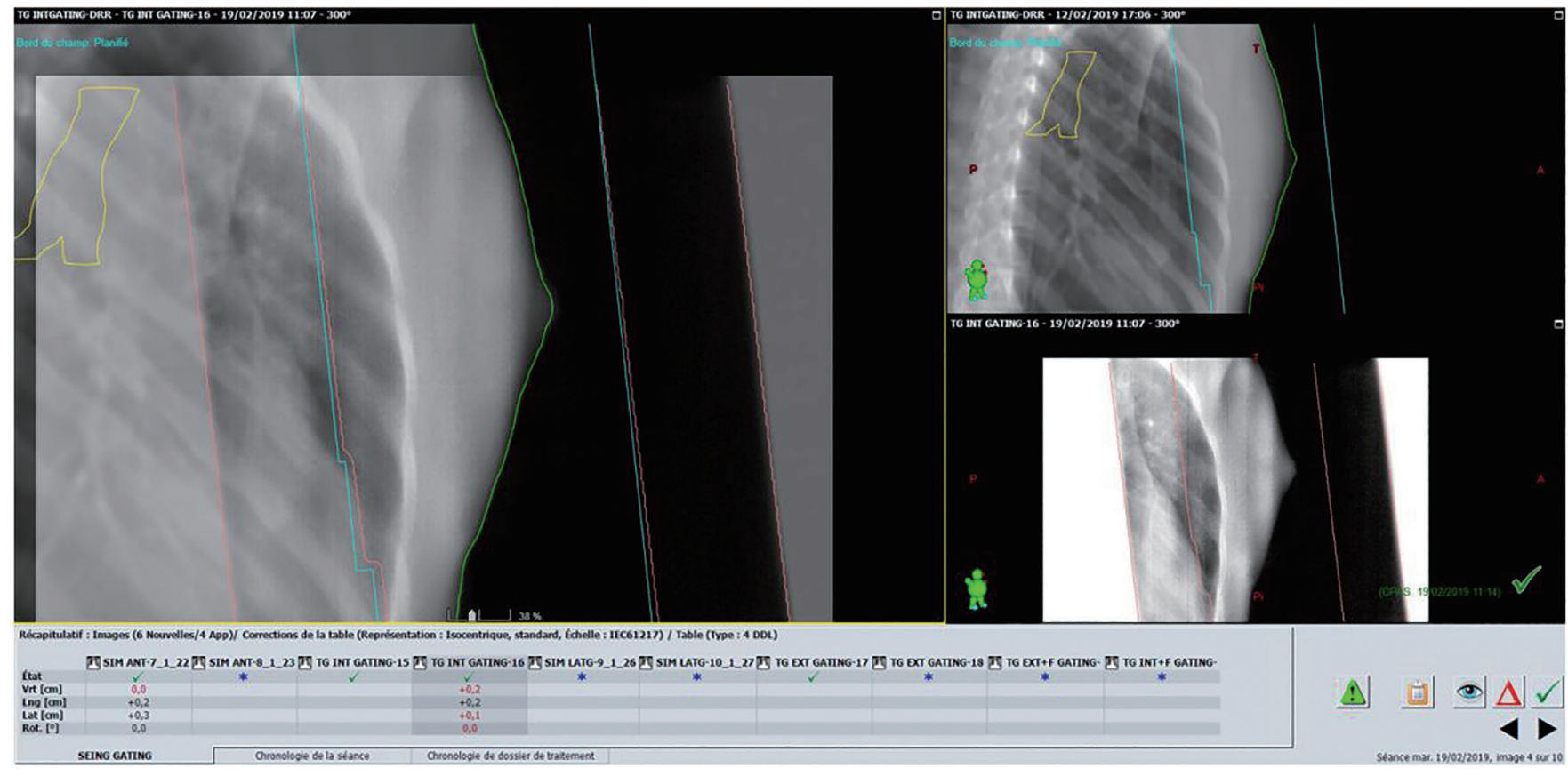

Figure 1 Screen capture of the repositioning imaging performed on a Clinac $2100^{\circledR}$, by double exposure for a patient irradiated on the left breast. The left part of the screen shows the matching obtained between the daily imaging and the reference one (digitally reconstructed radiograms, DRR), the right window shows the DRR (top) and the daily image (bottom). In the lower strip of the window are displayed the corrections to apply and the status of medical approval.

oncologists regarding this part of their prescription in the realization of treatments. Thus, they are able to make any decision to alleviate, if deemed necessary, this over dosage. This information may be critical, in particular, for situations as for re-irradiation. It is also of interest in a perspective of development of risk models of radiation-induced secondary cancers. Actually, this exposure source, if neglected, would lead to overestimate the role of therapeutic irradiation itself in epidemiological analysis of this risk.

\section{Methods}

\section{Radiotherapy plans}

For reasons of homogeneity in order to limit biases, only one frequent radiotherapy indication was chosen: postoperative mammary irradiation. Patients received either 62.1 or 50.6 Gy in 23 fractions, 5 fractions a week, according respectively to the use or not of a concomitant boost in resection site of 11.5 Gy in 0.5 Gy per fraction. Almost all patients were treated likewise. Repositioning images were recorded for 3 accelerators, 1 Elekta ${ }^{\circledR} \mathrm{SLi}$ and 2 Varian ${ }^{\circledR}$ machines (Clinac $600^{\circledR}$ and $2100^{\circledR}$ ) commissioned in 2002, 2010 and 2006, respectively.

Images were realized in double exposure (Figure 1) with a first image corresponding exactly to the treatment field and then an enlarged second one, by opening the collimator, to visualize the anatomical environment and control the positioning. This procedure was applied weekly for at least two incidences per patient and repeated if necessary, in case of positioning difficulties. A normal standard procedure included 4 images in two pairs of double exposures, which is counted as 2 images. Each image of a pair (field "open" + field "closed") was made with the same number of MU for a given machine.

For dose calculation in a small subset of patients, the real radiotherapy plans were used to estimate the delivered dose using imaging processing as described above.

\section{Characteristics of the electronical portal imaging devices (EPID)}

\section{EPID of Elekta SLi ${ }^{\oplus}$}

The Elekta $\mathrm{SLi}^{\circledR}$ was equipped with an $\mathrm{iViewGT}^{\circledR}$ system associating a $40 \times 40 \mathrm{~cm}^{2}$ inorganic scintillator detector with 
a covering copper plate. The same principle was used for the Clinacs (10). The amorphous silicon active detection surface (a-SI) was composed of a $1,024 \times 1,024$ photodiodetransistor associations matrix.

The spatial resolution was given by a f50 $=0.28 \mathrm{~mm}^{-1}$. The sensitivity of the detector was relatively low and the machine required at least 3 monitor units (MU) per image otherwise mentioning "detector underexposed: no image" and producing no image. The device was positioned at a fixed distance from the couch and was only movable laterally and longitudinally ( $\mathrm{x}$ and $\mathrm{y}$ ), which constrained the spatial resolution and the dose required to obtain an image.

The acquired images were then compared to the reference DRRs (digitally reconstructed radiograms), from the dosimetry CT scan, on an ancillary console thanks to the iView ${ }^{\circledR}$ system, however without the possibility of image matching for comparison purpose.

\section{EPID of Clinac $2100^{\circledR}$ and Clinac $600^{\circledR}$}

Both Clinac's had a $30.1 \times 40.1 \mathrm{~cm}^{2}$ semiconductor cassette of the same amorphous silicon composition (11). Their pixel matrices were $512 \times 384$ with a pixel size of $0.784 \mathrm{~mm}$ for the Clinac $600^{\circledR}$, and $1,024 \times 768$ and $0.392 \mathrm{~mm}$ pixels for the Clinac $2100^{\circledR}$.

The EPID of the Clinac $600{ }^{\circledR}$ had a contrast resolution of $0.2 \%$ for $6 \mathrm{MV}$, and a spatial resolution of $\mathrm{f} 50=0.44 \mathrm{~mm}^{-1}$. For the Clinac $2100^{\circledR}$ the resolution in contrast was the same, and the spatial resolution was $f 50=0.50 \mathrm{~mm}^{-1}$. The detector sensitivity required a minimum of $1 \mathrm{MU} / \mathrm{image}$ for the Clinac $2100^{\circledR}$ and $2 \mathrm{MU} / \mathrm{image}$ for the Clinac $600^{\circledR}$.

The device was mounted on a movable arm in the 3 dimensions of space $\left(\mathrm{R}-\mathrm{ARM}^{\circledR}\right)$. The patient-detector distance was therefore adjustable which allowed a better resolution for an equivalent dose delivery. The images were acquired by "Double exposure before irradiation" mode using Varian's IAS ${ }^{\circledR}$ system and assessed with Offline Review $^{\circledR}$ software.

\section{Images and data acquisitions}

The current practice over the whole time of the data collection was to perform one image of each beam on the first treatment day, and then two opposite beams weekly. In addition, in case of difficulties for repositioning, daily control images could be done. The typical number was therefore 5 weeks $\times 2$ images + the initial images of each beam ( 6 in general due to mixed energies, boost and wedged fields) plus additional images for difficulties. This totalized about 20 to 25 images per patient treatment course.

The images were automatically collected by a software developed locally to extract acts and sessions from the patient (Oncology Information System, OIS) database (Varian ARIA ${ }^{\circledR}$ ).

The numbers of $M U$ for patient imaging were standardized but different according to the linac due to the sensitivity of the detector and the possibility of adjustment of the detector-patient distance, as discussed above.

The collection of data was done by the software that retrieved the control imaging activity codes for each patient, as mentioned above. Calculations were then performed by the same software according to formula (1). Radiation oncologists had access at any time to the cumulative imaging dose during treatment for each of their patients, and may demand, if they deemed it necessary, an adaptation of the treatment course of the patient.

This study did not require ethical approval nor informed consent since it has been performed with consolidated data of technical activity stored in the record-and-verify system of our department with no indications relative to the patients. Thus, the used data did not represent any more personal data according to the current legal definition of the French CNIL given in its deliberation No. 2018-155 of May 3rd, 2018 relative to the methodology of data management of processing of personal data carried out in the context of research not involving the human person in the field of health (MR-004).

\section{Calculation of the representative dose of imaging in reference conditions}

The calculation of doses, for a given patient, in reference conditions produced by the imaging procedures took into account the images number, the number of MU per image (MU/image) and the dose in Gy per MU [dose (Gy)/MU]. The reference conditions were $10 \mathrm{~cm} \times 10 \mathrm{~cm}$ beam at $5 \mathrm{~cm}$ depth, exactly the same of the daily checking of the beams, but also deemed well adapted to the average breast volume.

Thus, the imaging dose $\left(\mathrm{D}_{\text {image }}\right)$ is:

$$
\begin{aligned}
D_{\text {image }}(\text { Gy })= & (\text { images number }) \times(\text { MU per image }) \\
& \times(\text { dose in Gy per MU })
\end{aligned}
$$

The number of MU/image were $3 \mathrm{MU}, 2 \mathrm{MU}$ and $1 \mathrm{UM}$, respectively, for Elekta SLI ${ }^{\circledR}$, Clinac $600^{\circledR}$ and Clinac $2100^{\circledR}$. The ratio dose (Gy)/MU was 0.008 Gy in 
the reference conditions according to the IAEA, TRS-398 protocol (12).

The true ratio dose/MU was a specific value for each machine, monitored daily during the morning quality control of machines. The deviation of this ratio must not exceed $\pm 2 \%$ of the reference value. The calculation was made from the reference conditions.

\section{Comparison of representative imaging dose with calculated dose in treatment plan}

All the doses $\left(\mathrm{D}_{\text {image }}\right)$ recorded are those established under reference conditions. This is a standardized approximation allowing their automatic extraction of treatment session records for real-time information of the physicians. It is useful to compare these values with a representative set of "real" doses received by patients by establishing these doses from the treatment plan for a sample of patients. Thus, the dose at the center of the breast $\left(\mathrm{D}_{\text {center }}\right)$, at mid-depth and mid-thickness of the breast (ICRU reference point) delivered by the real imaging fields, was calculated with the treatment planning system and called $\left(\mathrm{D}_{\mathrm{TPS}}\right)$. The $\mathrm{D}_{\text {center }}$ $\approx \mathrm{D}_{\text {TPS }}$, was then compared with $\mathrm{D}_{\text {image }}$ the standardized representative imaging dose calculated by Eq. [1]. A sample of six patients $(n=6)$ has been selected in this purpose. Beside this comparison between $\mathrm{D}_{\text {image }}$ and $\mathrm{D}_{\text {center }} \approx \mathrm{D}_{\text {TPS }}$, it is important to mention that it remains a representative dose according to ICRU but not an accurate information of dose distribution as a HDV could be.

\section{Results}

\section{Reporting of imaging doses per patient}

For each patient, the standardized cumulative dose was registered, session after session, by a calculation tool associated to the patients' file in the record and verify software (Figure 2). The doses of all the ongoing treated patients were gathered and presented to their referent radiation oncologists once a week as a table including the name, surname, identification number of the patient, the name of the referent radiation oncologist, the identification of the treatment course, the number of cumulative images made for the patients and the standardized cumulative dose corresponding to these images with a color code attracting the attention when the dose was exceeding 0.4 Gy (Figure 3). This report is a decision tool for the MDs and could lead to a dose reduction by removing a session if deemed necessary.
Thus, the Figure 2 shows an example of the calculated imaging dose for a right breast tumor plan including a boost: two irradiated fields (external and internal) and two simulated fields for positioning. In this example, 4 images were made for the boost plan and 45 images for the breast plan leading to 49 images and a total cumulative standardized dose $\mathrm{D}_{\text {image }}=0.39 \mathrm{~Gy}$. The total dose was calculated according to Eq. [1] considering the dose per image as Dose (Gy)/MU $=0.008$ and $1 \mathrm{MU} /$ image for the Clinac 2100.

And the Figure 3 is an example of a weekly report of cumulative doses delivered per patient for a series of selected patients having different types of breast irradiation.

\section{Distribution of total delivered doses}

First, it is interesting to observe the distribution of total standardized doses delivered by our three devices.

The Figure 4 shows the total set of standardized dose results for the whole population according to the type of machine/portal imaging used for these breast cancer population. It can be seen that, the machine/device is obviously the most important parameter as their differences of sensitivities may anticipate it.

The Table 1 shows the number of patients ordered by dose bins, by steps of 1 Gy. It can be seen that, the doses exceeding 2 Gy represent 15 patients (about 1\% of the total population) and only happen with the Elekta $\mathrm{SLi}^{\circledR}$. The maximum dose in class (4 to $5.05 \mathrm{~Gy}$ ) was $5.02 \mathrm{~Gy}$ which is $10 \%$ of the prescribed dose (50 Gy) for this indication.

Finally, the Table 2 shows the total cumulative imaging dose (Gy) for all patients and the three used machines as average (with standard deviation), minimal and maximal which are respectively $(0.21 \pm 0.16 ; 0.03$ to 1.7$),(0.24 \pm 0.16$; 0.06 to 1.0$)$ and $(0.69 \pm 0.39 ; 0.09$ to 5.02$)$ for the Clinac 2100, Clinac 600 and Elekta SLi. The differences are statistically significant between the three machines. These differences were not related to the length or the total dose delivered since almost all patients received 23 fractions. These differences were related to complexity of the treated volume and/or the difficulties to reposition some patients.

\section{Comparison of a sample of calculated doses with the standardized doses}

To assess the consistency of the standardized doses with a more personalized estimation of the dose, namely the 


\begin{tabular}{|c|c|c|c|c|c|c|c|}
\hline \multicolumn{8}{|c|}{ Estimation of the doses delivered by the control imaging } \\
\hline Last Name & First Name & Date Of Birth & Patient Id & Course Id & Clinical Status & DR & Start Date \\
\hline$x x x x x$ & $\operatorname{xxxxx}$ & $x x / x x / x x x$ & $x x x x x x x$ & R Breast & Completed & C50.9 & $x x / x x / x x x x$ \\
\hline \multicolumn{8}{|c|}{ Out of field cumulative imaging doses per session (any machine) } \\
\hline Machine & Course Id & \multicolumn{2}{|c|}{ Dose(Gy)/MU } & MU/Image & num images & \multicolumn{2}{|r|}{ Total Dose (Gy) } \\
\hline Clinac2100 & R Breast & \multicolumn{2}{|c|}{0.008} & 1 & 49 & \multicolumn{2}{|r|}{0.3920} \\
\hline \multicolumn{8}{|c|}{ Out of field cumulative imaging doses per beam } \\
\hline Machine & Beam Id & \multicolumn{2}{|c|}{ Dose(Gy)/MU } & MU/Image & num images & \multicolumn{2}{|r|}{ Total Dose (Gy) } \\
\hline Clinac2100 & EXT & \multicolumn{2}{|c|}{0.008} & 1 & 18 & \multicolumn{2}{|r|}{0.1440} \\
\hline Clinac2100 & INT & \multicolumn{2}{|c|}{0.008} & 1 & 22 & \multicolumn{2}{|r|}{0.1760} \\
\hline Clinac2100 & SIM ANT & \multicolumn{2}{|c|}{0.008} & 1 & 5 & \multicolumn{2}{|r|}{0.0400} \\
\hline Clinac2100 & SIM LAT R & \multicolumn{2}{|c|}{0.008} & 1 & 4 & \multicolumn{2}{|r|}{0.0320} \\
\hline \multicolumn{8}{|c|}{ Out of field cumulative imaging doses per plan } \\
\hline Machine & Plan Setupld & \multicolumn{2}{|c|}{ Dose (Gy)/MU } & MU/Image & num images & \multicolumn{2}{|r|}{ Total Dose (Gy) } \\
\hline Clinac2100 & Boost QSE R & \multicolumn{2}{|c|}{0.008} & 1 & 4 & \multicolumn{2}{|r|}{0.0320} \\
\hline Clinac2100 & R Breast & \multicolumn{2}{|c|}{0.008} & 1 & 45 & \multicolumn{2}{|r|}{0.3600} \\
\hline
\end{tabular}

Figure 2 Example of table as issued from the Record and verify system a case of left breast cancer. After the identification lines, the first line gives the cumulative of all imaging doses, the second part of the table details by beam the images made and the resulting doses, the last part of the table gives the cumulative dose per treatment course (main volume vs. boost). For this case, an additional cumulative dose of 0.4 Gy was delivered by the imaging procedure. The doses were calculated according to Eq. [1]. Two irradiated fields external and internal and two simulated fields for positioning were used. EXT, external; INT, internal; SIM, simulated fields; ANT, anterior; LAT, lateral; R, right; num images, number images; MU, monitor units.

\begin{tabular}{|c|c|c|c|c|c|c|c|c|}
\hline Patient name & Patient Id & Physician & Course ID & Machine & Number of Images & Dose (Gy) & Color code & Dose (Gy) \\
\hline A & $x x x x x$ & 1 & R Breast & Clinac2100 & 49 & 0.39 & & Dose $<0.4$ \\
\hline B & $x x x x x$ & 2 & R Breast & Clinac2100 & 31 & 0.25 & & \\
\hline C & $x x x x x$ & 1 & L Breast & Clinac2100 & 44 & 0.35 & & $0.4<$ Dose $<0.8$ \\
\hline D & $x x x x x$ & 2 & L Wall & Clinac2100 & 31 & 0.25 & & \\
\hline E & $x x x x x$ & 1 & $\mathrm{R}$ Breast & Clinac2100 & 30 & 0.24 & & $0.8<$ Dose $<1.2$ \\
\hline $\mathrm{G}$ & $x x x x x$ & 2 & L Breast & Clinac2100 & 30 & 0.24 & & $1.2<$ Dose \\
\hline $\mathrm{H}$ & $x x x x x$ & 2 & L Wall & Clinac2100 & 50 & 0.40 & & \\
\hline
\end{tabular}

Figure 3 Weekly table summarizing cumulative doses delivered per patient. The first and second columns correspond respectively to the name and identification number of the patient. The following columns correspond respectively to the radiation oncologist responsible for the patient, the name of the treatment course, the number of cumulative images made for the patients and the cumulative dose of the imaging. The color code facilitates the reading and warns about the doses received by imaging when they start to become important. R, right; L, left. 


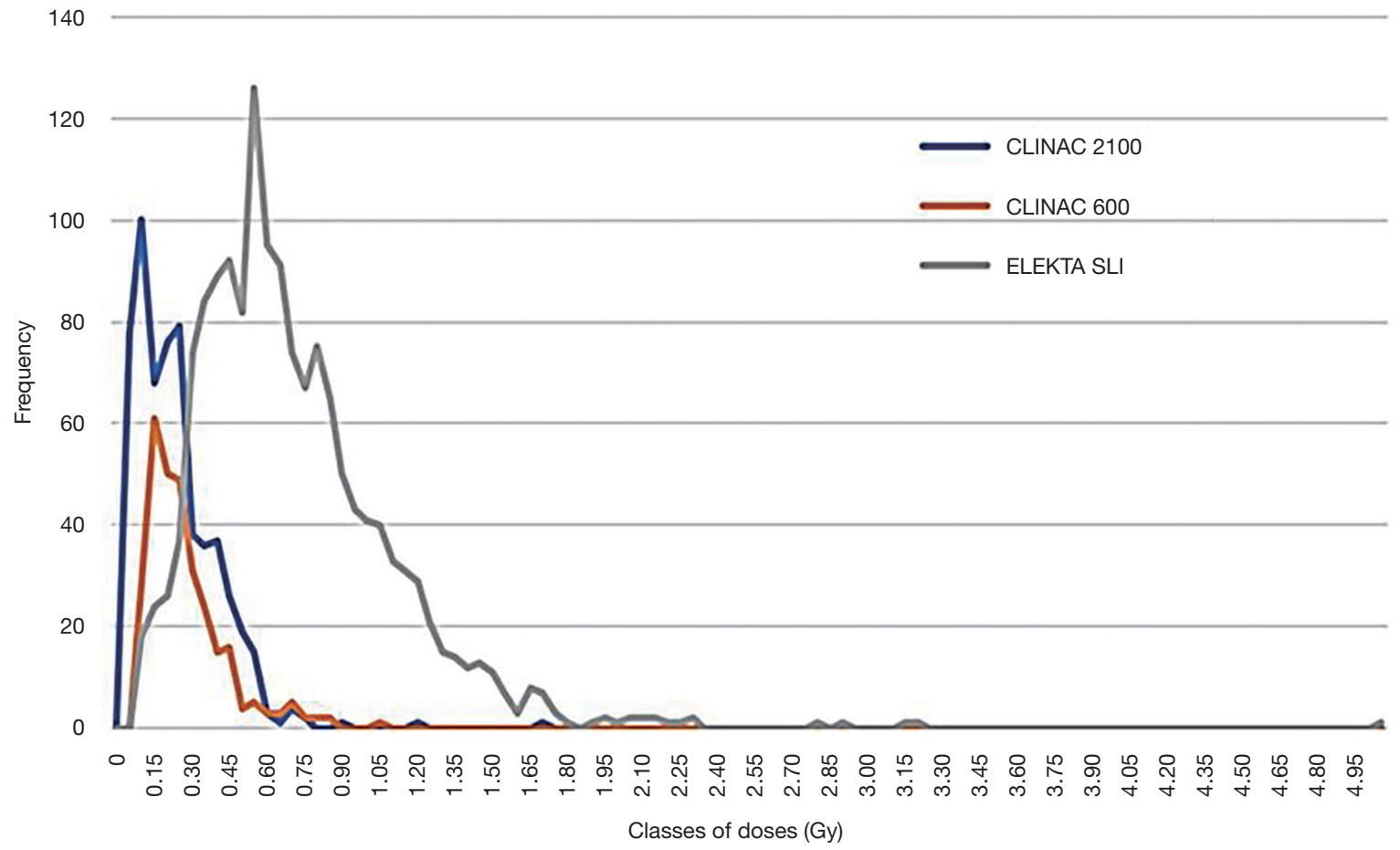

Figure 4 The classes of standardized dose from the portal imaging for the three machines performed in the context of the breast treatments according to the treatment apparatus, for 10 years of activity. The curves represent the doses distribution histograms by bins of 0.1 Gy. There is an almost equal peak for Clinac $2100^{\circledR}$ and Clinac $600^{\circledR}$ at the respective doses of 0.1 and 0.15 Gy. The highest doses are achieved by the Elekta SLi ${ }^{\circledR}$ peaking at $0.55 \mathrm{~Gy}$. Note the spreading of the data ranges, with the largest area under the curve for Elekta SLi ${ }^{\circledR}$ then the Clinac $600^{\circledR}$ and finally Clinac $2100^{\circledR}$.

Table 1 Number of patients ordered by dose bins, by steps of 1 Gy

\begin{tabular}{lccc}
\hline \multirow{2}{*}{$\begin{array}{l}\text { Dose intervals } \\
\text { (Gy) }\end{array}$} & \multicolumn{3}{c}{ Number of patients } \\
\cline { 2 - 4 } & ${\text { Clinac } 2100^{\circledR}}$ & ${\text { Clinac } 600^{\circledR}}^{\text {Elekta SLi }^{\circledR}}$ \\
\hline $0-1$ & 582 & 300 & 1,253 \\
$1-2$ & 2 & 0 & 252 \\
$2-3$ & 0 & 0 & 12 \\
$3-4$ & 0 & 0 & 2 \\
$4-5.05$ & 0 & 0 & 1 \\
\hline
\end{tabular}

real calculation of the dose delivered to the patient in the real condition of imaging, a sample of representative patients has been chosen and for each one the calculation has been performed using the TPS. The Figure 5 gives as a boxplot the results for a series of 6 patients per Linac distributed over time and representative of different treatment situations and not extreme in terms of anatomical conditions.
In summary, the estimated differences between the dose calculated by TPS ( $\mathrm{D}_{\text {TPS }}$ ) with the imaging dose in standardized $\left(\mathrm{D}_{\text {image }}\right)$ condition as well as with the most representative doses at the ICRU point $\left(\mathrm{D}_{\text {center }}\right)$ differs by less than $7 \%$ (from $-3.2 \%$ to $+7 \%$ depending on the machine).

We can thus consider that the $\mathrm{D}_{\text {image }}$ is correctly representing the dose delivered by the repositioning imaging in the breast. It should be noted that these $\mathrm{D}_{\text {TPS }}$ and $\mathrm{D}_{\text {center }}$ doses are also very close to the maximum dose received in the thinnest region of the breast, that is to say the nipple region.

\section{Discussion}

This monocentric work, which represents the exhaustive record of portal doses over the course of 10 years of activity [2008-2018] at the University Hospital of Grenoble, made it possible to compare three machines in one of the most frequent activities of a radiotherapy department: postoperative breast irradiation. 
Table 2 Presentation for breast irradiations of the whole patient cohorts, the average doses with standard deviation as well as minimal and maximal doses. These doses take account of the total number of images made per patient

\begin{tabular}{lcccc}
\hline Machines & Patients & Average dose \pm SD (Gy) & Minimal dose (Gy) & Maximal dose (Gy) \\
\hline Clinac 2100 & 584 & $0.21 \pm 0.16$ & 0.03 & 1.7 \\
Clinac 600 & 300 & $0.24 \pm 0.16$ & 0.06 & 1.0 \\
Elekta SLi & 1,519 & $0.69 \pm 0.39$ & 0.09 & 5.02 \\
\hline
\end{tabular}

SD, standard deviation. The $P$ value obtained by the Student test were $<0.005$ showing significant differences between the three machines.

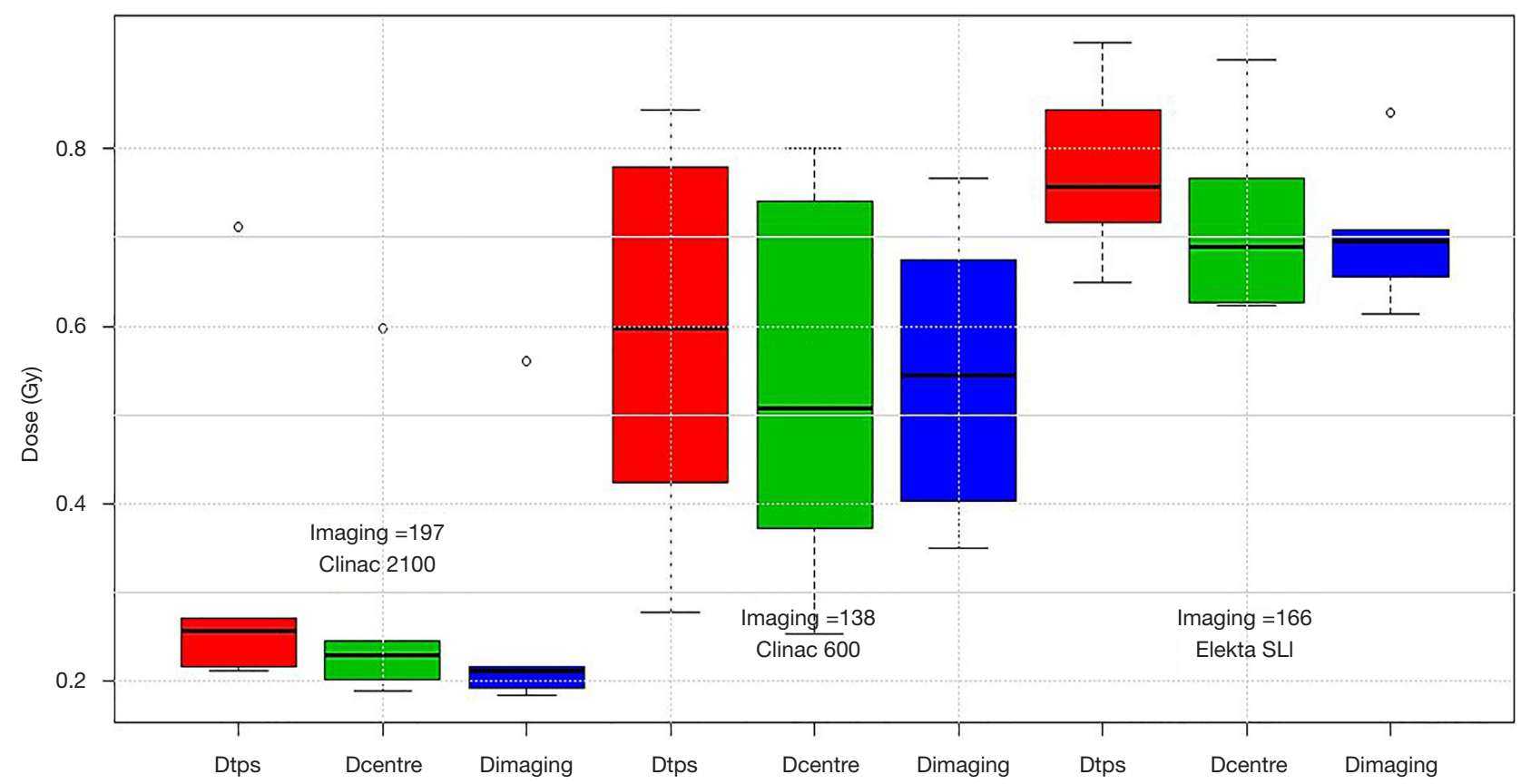

Figure 5 The case-specific data of the total imaging dose values for real and representative treatments on each machine, calculated using three methods: (I) the total maximum dose as calculated by the TPS which takes account of the maximum dose value in the imaged volume which is generally the nipple region $\left(\mathrm{D}_{\mathrm{TPS}}\right)$; (II) the total dose in the center of the breast $\left(\mathrm{D}_{\text {center }}\right)$ represented by the dose at mid-depth and mid-thickness of the breast (ICRU reference point) which is the most representative of the dose delivered; (III) the total imaging dose ( $\mathrm{D}_{\text {imaging }}$ ) in standardized condition (beam of $10 \times 10 \mathrm{~cm}^{2}$ at $5 \mathrm{~cm}$ depth). The average Dcenter, presented in green is shown to be slightly higher than the calculated Dimaging ("physician-reported") for the Clinac $2100^{\circledR}$ and slightly lower for the Clinac $600^{\circledR}$ and the Elekta SLi ${ }^{\circledR}$. The box plot represent from top to bottom either the extreme or maximum values, the $75^{\text {th }}$ percentile, the median, the $25^{\text {th }}$ percentile and the minimum values.

\section{Control and repositioning imaging}

Control and repositioning imaging are a central point in a radiotherapy service because of its necessity and importance for the smooth running of a treatment. The doses delivered by imaging vary considerably according to the sensitivity of the detector, and somehow also the age of the material, which determines the dose necessary to produce an interpretable image.

This study highlights the differences between the two brands of devices studied. These differences could however have been increased by bias of practice, i.e., the fact that treatments with all regional volumes (axilla-supraclavicular and MIC, in addition to the breast) were more often 
performed with the most dosing device, for simple reasons for organization to optimize the time of use of other linacs in favor of intensity-modulated radiotherapy (IMRT).

This study shows that the use of portal imaging, which has historically followed high-energy film imaging (gamma radiography), can represent a significant dosimetric contribution. In total, for an average treatment course, it can range from less than one gray to more than 5 Gy. In the latter case, this represents approximately $10 \%$ of the prescribed dose, which greatly exceeds the dosimetric level of uncertainties recommended elsewhere for the treatment plans validation. This considerable difference can be further increased by systematic daily practice, at each session, of these controls, which has not been the case here but which can potentially multiply by 4 or 5 the median doses reported in this study. However, it is expected that they can be identified and possibly taken into account in the treatment course.

Since this dosimetric information was not included in the treatment plan calculation, this statement resulted from an independent procedure, made after the treatment plan. Moreover, this dosimetric information represents an approximation insofar as it concerns a volume slightly larger than the treated volume (due to double exposure) without it being exactly specified and measured about what is contained in this enlarged volume. Indeed, it is as shown in Figure 1 on the one hand an increase in fall off, without consequences for the patient, and on the other hand an increase in the volume of pulmonary and cardiac irradiation, during these imaging, as well as the contralateral breast. It should also be mentioned that the dosimetric characterization of the images was using the MU values measured in standardized conditions (use of a homogeneous phantom, equivalent water density and beam opening of $10 \times 10 \mathrm{~cm}^{2}$, measured at $5 \mathrm{~cm}$ depth), which are different from the actual imaging situation. Both conditions have opposite effects; the first tends to overestimate the dose and the second to underestimate it.

The comparison of these reference doses with the calculated values from the own dosimetry of a series of patients, for each device, shows that this approximation remains within a range of $-2 \%$ to $+7 \%$. This approximation therefore seems acceptable to us for an overall survey work that did not deal with this question on a case-by-case basis, and that did not make the decision to plan this dose contribution as part of the individual treatment planning of each patient.

Similar works that have already been published, concerning portal imaging by EPID generally show either similar doses in MU per image or cumulative delivered doses of the order of the gray as shown in Table 3.

\section{Reporting imaging dose}

The recorded values were only the standardized doses calculated from $\mathrm{MU}$, and in all cases delivered in high energy photons (6 MV), which most of the time came out with the picture of the very beams of treatment. This type of imaging in breast radiotherapy had more a quality assurance value, especially for lung protection, than for primary target repositioning, the latter being carried out clinically with external markers (laser, checking source-skin distance, fall off). It was therefore a question of checking the beam eye view rather than the position. The imaging beams, always mimicking the exact beams contributing to the treatment, with a similar energy, one could consider that the imaging dose (known at $5 \mathrm{~cm}$ depth) was comparable in volume distribution to the dose of treatment and was delivered to the same targets. It can therefore be considered that the addition of the imaging doses to the treatment doses was an acceptable approximation.

\section{Impact of image device on the delivered dose}

As presented in Figure 4 and Table 2 a significant difference was observed between the three machines. This is explained by the larger dose spreading of the $\mathrm{SLi}^{\circledR}$, but also the presence of a second dose peak for the Clinac $2100^{\circledR}$. It should also be noted that the area under the dose curves of the 2100 and 600 are different with an upper area for the Clinac $2100^{\circledR}$. As for the Elekta $\mathrm{SLi}^{\circledR}$ we may notice a peak well superior to those of both Clinac. This is also notable in comparison with the standard deviations which are direct indicators of the larger dose spread for the Elekta $\mathrm{SLi}^{\circledR}$ and Clinac $2100^{\circledR}$ and more reduced for the Clinac $600^{\circledR}$.

The differences in imaging doses delivered by the different devices result first from the differences in sensitivity of the detectors. Indeed, as previously announced, the detectors are of different compositions and had different positioning between Clinac and Elekta SLi ${ }^{\circledR}$ inducing different MU's needs. Added to this, was the insufficient functionality of the $\mathrm{SLi}^{\circledR}$ image comparison tool. This one did not allow image matching between DRR and PI of the day, which can lead to the realization of several images for successive corrections.

Unexpectedly, the most sensitive machine, the Clinac 
Table 3 Synthesis of the literature listing reports authors including the patient numbers, tumor locations, imaging techniques and monitor units numbers, cumulative doses per patient, and effective doses when reported

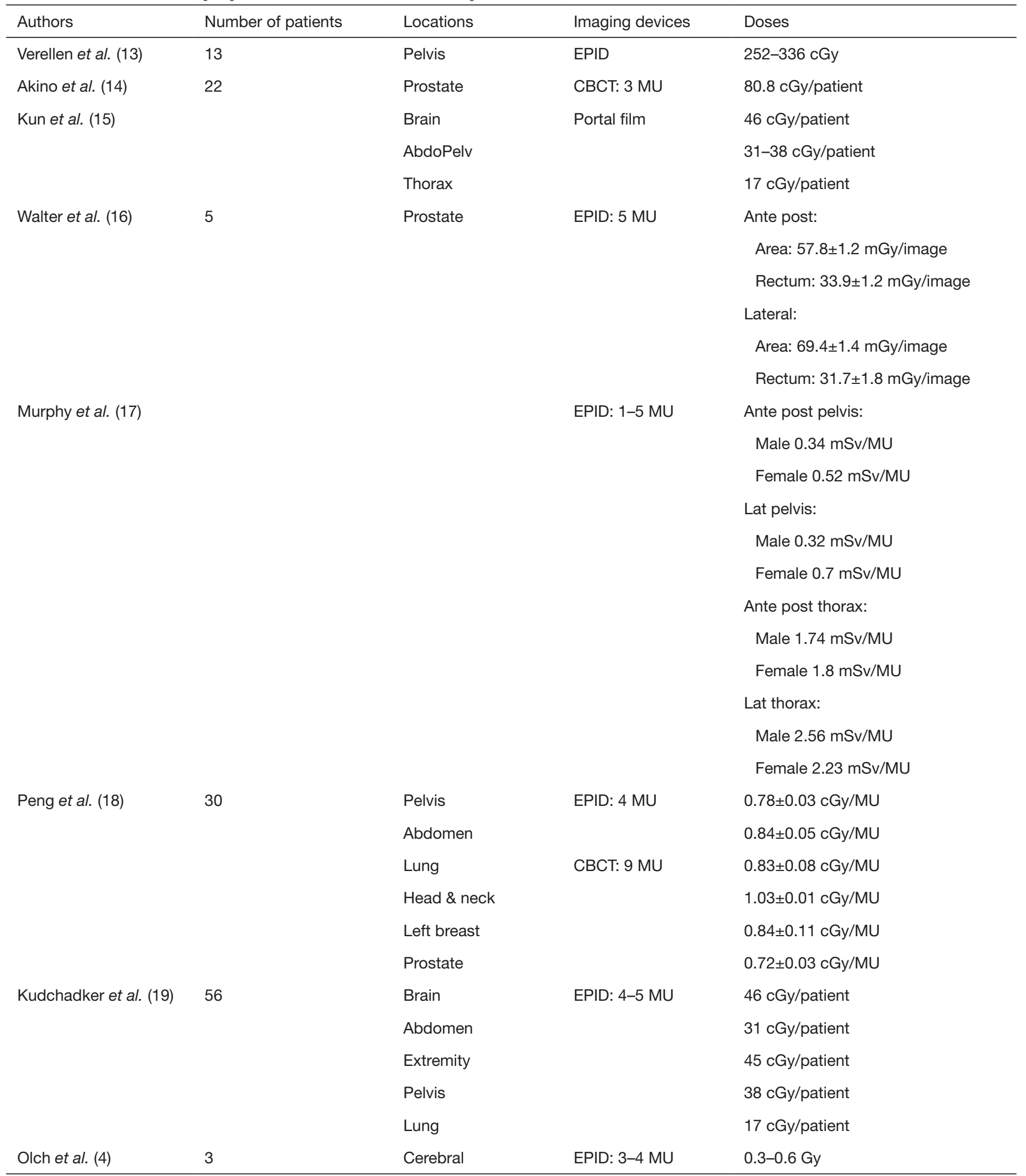

MU, monitor units; EPID, electronical portal imaging devices; CBCT, cone beam computed tomography; lat, lateral; Ante, anterior; Post, posterior; AbdoPelv, abdomen-pelvis. 
2100 was associated with more dose per patient than for the Clinic 600. This difference can be explained by the presence of breast treatments requiring or not the irradiation of the supra-and sub clavicular or axillary lymph nodes, or the internal mammary chain. These treatments required more PI repositioning imaging. Moreover, some irradiations were done in electron beams or with $18 \mathrm{MV}$ photons, being present only on the Clinac $2100^{\circledR}$. Thus, the simplest irradiations of the mammary gland alone were mostly performed with the Clinac $600^{\circledR}$.

Ding et al. (20), showed that the use of 2.5 MV beam for portal imaging is able to reduce by half the dose delivered to deep organs as demonstrated by MC simulation. However, in current practice this type of beam remains unusual, as only few linacs if any are providing such specific energy, presently new equipments are mostly providing CBCT modalities with $\mathrm{kV}$ beams.

\section{Consideration of imaging dose in modeling dose response}

This work, which concerns practices and devices that are no longer in use today, however, highlights a dosimetric contribution that is generally ignored and not taken into consideration in the benchmarks of good clinical practice in terms of doses to prescribe. Thus, the subsequent interpretation of epidemiological events related to the irradiation of these patients, in ignorance of these dose increments provided by imaging, would overestimate the toxic impact of therapeutic irradiation, either deterministic or stochastic (21). The sensitivity of radiobiological models requires, for their reliable use, to have particularly accurate dosimetric input data (22). This work can help to adjust the dose values to be considered.

During the practice of these imaging procedures, the awareness of the doses delivered led us to distribute to the radiation oncologists a weekly summary of the cumulated doses by patient related to the control imaging. They were given the opportunity to take corrective measures which, for example, could be the removal of the last treatment session. Moreover, these dose readings can also bring out other information such as the difficulty of repositioning a given patient (specific increase in the dose of a patient) or even the degradation of the receptor (systematic increase of doses delivered).

Some centers are able to go further and propose the calculation of a new treatment plan, taking into account the doses delivered by the imaging. Current treatment planning software will progressively move in this direction, particularly in the context of the race for dosimetric accuracy of adaptive radiotherapy.

\section{Conclusions}

The imaging dose using EPID from three machines were compared and analyzed for breast treatment. A significant dose difference was observed since the dose/image depend on the delivered dose/MU. To acquire an image 1, 2 or $3 \mathrm{MU}$ were used according to the different Linac systems. Consequently, an important average cumulative (0.2-0.7 Gy) can be delivered. The imaging doses could be presented weekly to radiation oncologist for patient radiation protection purpose and should be registered to be considered in a possible study of long-term deterministic and stochastic risks.

\section{Acknowledgments}

Authors are grateful to Y. Kefs for his help in reviewing the manuscript and the translation.

Funding: None.

\section{Footnote}

Conflicts of Interest: All authors have completed the ICMJE uniform disclosure form (available at http://dx.doi. org/10.21037/qims-19-1031). Dr. AC serves as an unpaid editorial board member of Quantitative Imaging in Medicine and Surgery. The other authors have no conflicts of interest to declare.

Ethical Statement: The authors are accountable for all aspects of the work in ensuring that questions related to the accuracy or integrity of any part of the work are appropriately investigated and resolved. This study did not require ethical approval nor informed consent since it has been performed with consolidated data of technical activity stored in the record-and-verify system of our department with no indications relative to the patients. Thus, the used data did not represent any more personal data according to the current legal definition of the French CNIL given in its deliberation No. 2018-155 of May 3rd, 2018 relative to the methodology of data management of processing of personal data carried out in the context of research not involving the human person in the eld of health (MR-004).

Open Access Statement: This is an Open Access article 
distributed in accordance with the Creative Commons Attribution-NonCommercial-NoDerivs 4.0 International License (CC BY-NC-ND 4.0), which permits the noncommercial replication and distribution of the article with the strict proviso that no changes or edits are made and the original work is properly cited (including links to both the formal publication through the relevant DOI and the license). See: https://creativecommons.org/licenses/by-nc-nd/4.0/.

\section{References}

1. Principle of radioprotection doc. $\mathrm{ASN} \mathrm{n}^{\circ} 2$. Available online: https://www.asn.fr/Informer/Publications/ Fiches-d-information-du-public/Les-principes-deradioprotection (access on May 11th, 2019).

2. Accreditation criteria of INCa for external radiotherapy practice. Available online: https://www.e-cancer. fr/content/download/58314/531708/file/criteres_ radiotherapie_160608.pdf (access on May 11th, 2019).

3. Peiffert D, Simon JM, Eschwege F. Epinal radiotherapy accident: passed, present, future. Cancer Radiother 2007;11:309-12.

4. Olch AJ, Geurts M, Thomadsen B, Famiglietti R, Chang EL. Portal imaging practice patterns of children's oncology group institutions: Dosimetric assessment and recommendations for minimizing unnecessary exposure. Int J Radiat Oncol Biol Phys 2007;67:594-600.

5. Ding G, Munro P. Minimizing IGRT Imaging Exposures: KV Radiograph Vs. KV-CBCT Vs. MV Portal Images. Med Phys 2012;39:3661.

6. Harrison RM, Wilkinson M, Shemilt A, Rawlings DJ, Moore M, Lecomber AR. Organ doses from prostate radiotherapy and associated concomitant exposures. Br J Radiol 2006;79:487-96.

7. Ding GX, Alaei P, Curran B, Flynn R, Gossman M, Mackie TR, Miften M, Morin R, Xu XG, Zhu TC. Image guidance doses delivered during radiotherapy: Quantification, management, and reduction: Report of the AAPM Therapy Physics Committee Task Group 180. Med Phys 2018;45:e84-99.

8. Wang X, Du W, Smith SA, Stovall M, Buchholz TA, Salehpour M. The radiation exposure from portal images during the course of breast radiotherapy. Am J Clin Oncol 2008;31:345-51.

9. Harrison RM, Wilkinson M, Rawlings DJ, Moore M. Doses to critical organs following radiotherapy and concomitant imaging of the larynx and breast. Br J Radiol 2007;80:989-95.
10. State of the art of transit dosimetry for in vivo dosimetry control in radiotherapy in France. Report number PSESANTE/SER/2018-00003. Available online: https:// www.irsn.fr/FR/expertise/rapports_expertise/Documents/ radioprotection/IRSN-PSE-SANTE-SER-2018-00003_ Dosimetrie-de-transit.pdf

11. PortalVision Advanced imaging. Varian Medical System. Available online: http://www.equiphos.com/wp-content/ uploads/2015/05/PortalVision_Advanced-Imaging_ Specs_10087B_0312.pdf (access on 11 May 2019).

12. Technical Reports Series No. 398. Absorbed Dose Determination in External Beam Radiotherapy: An International Code of Practice for Dosimetry based on Standards of Absorbed Dose to Water. International atomic energy agency, Vienna, 2000. Available online: https://www-pub.iaea.org/MTCD/publications/PDF/ TRS398_scr.pdf

13. Verellen D, De Neve W, Van den Heuvel F, Coghe M, Louis O, Storme G. On-line portal imaging: image quality defining parameters for pelvic fields. A clinical evaluation. Int J Radiat Oncol Biol Phys 1993;27:945-52.

14. Akino Y, Koizumi M, Sumida I, Takahashi Y, Ogata T, Ota S, Isohashi F, Konishi K, Yoshioka Y. Megavoltage cone beam computed tomography dose and the necessity of reoptimization for imaging dose-integrated intensitymodulated radiotherapy for prostate cancer. Int J Radiat Oncol Biol Phys 2012;82:1715-22.

15. Kun LE, Beltran C. Radiation therapy for children: evolving technologies in the era of ALARA. Pediatr Radiol 2009;39:S65-70.

16. Walter C, Boda-Heggemann J, Wertz H, Loeb I, Rahn A, Lohr F, Wenz F. Phantom and in-vivo measurements of dose exposure by image-guided radiotherapy (IGRT): MV portal images vs. $\mathrm{kV}$ portal images vs. cone-beam CT. Radiother Oncol 2007;85:418-23.

17. Murphy MJ, Balter J, Balter S, BenComo JA Jr, Das IJ, Jiang SB, Ma CM, Olivera GH, Rodebaugh RF, Ruchala KJ, Shirato H, Yin FF. The management of imaging dose during image-guided radiotherapy: report of the AAPMTask Group 75. Med Phys 2007;34:4041-63.

18. Peng LC, Jack Yang CC, Sim S, Weiss M, Bielajew A. Dose comparison of megavoltage cone-beam and orthogonal-pair portal images. J Appl Clin Med Phys 2006;8:10-20.

19. Kudchadker RJ, Chang EL, Bryan F, Maor MH, Famiglietti R. An evaluation of radiation exposure from portal films taken during definitive course of pediatric radiotherapy. Int J Radiat Oncol Biol Phys 
2004;59:1229-35.

20. Ding GX, Munro P. Characteristics of 2.5 MV beam and imaging dose to patients. Radiother Oncol 2017;125:541-7.

21. International Commission on Radiological Protection. ICRP Publication 60; Pergamon, Press, Oxford, 1991.

22. Chaikh A, Docquière N, Bondiau PY, Balosso J. Impact

Cite this article as: Kefs S, Giraud JY, Naud J, Henry I, Gabelle-Flandin I, Balosso J, Chaikh A, Verry C. Doses delivered by portal imaging quality assurance in routine practice of adjuvant breast radiotherapy worth to by monitored and compensated in some cases. Quant Imaging Med Surg 2021;11(8):3481-3493. doi: 10.21037/qims-19-1031 of dose calculation models on radiotherapy outcomes and quality adjusted life years for lung cancer treatment: do we need to measure radiotherapy outcomes to tune the radiobiological parameters of a normal tissue complication probability model? Transl Lung Cancer Res 2016;5:673-80. 